\title{
Bone density and neuromuscular function in older competitive athletes depend on running distance
}

\author{
U. Gast • D. L. Belavý • G. Armbrecht • K. Kusy • H. Lexy • \\ R. Rawer • J. Rittweger • K. Winwood • J. Zieliński • D. Felsenberg
}

Received: 21 June 2012 / Accepted: 29 November 2012 /Published online: 15 December 2012

(C) International Osteoporosis Foundation and National Osteoporosis Foundation 2012

\begin{abstract}
Summary Individuals who are involved in explosive sport types, such as 100-m sprints and long jump, have greater bone density, leg muscle size, jumping height and grip strength than individuals involved in long-distance running. Introduction The purpose of this study is to examine the relationship between different types of physical activity with bone, lean mass and neuromuscular performance in older individuals.
\end{abstract}

U. Gast • D. L. Belavý • G. Armbrecht • H. Lexy • D. Felsenberg Centre for Muscle and Bone Research, Charité

Universitätsmedizin Berlin, Hindenburgdamm 30,

12203 Berlin, Germany

K. Kusy $\cdot$ J. Zieliński

Department of Athletics, Eugeniusz Piasecki University

School of Physical Education, ul. Królowej Jadwigi, 27/39,

61-871 Poznań, Poland

R. Rawer

Novotec Medical GmbH, Pforzheim, Germany

J. Rittweger

German Aerospace Center (DLR),

Institute of Aerospace Medicine, Linder Höhe,

51147 Cologne, Germany

\section{J. Rittweger}

Institute for Biomedical Research into Human Movement and Health, Manchester Metropolitan University, Manchester,

England, UK

K. Winwood

Institute for Performance Research,

Manchester Metropolitan University, Crewe Campus,

Manchester, England, UK

U. Gast $(\square)$

Zentrum für Muskel- und Knochenforschung,

Charité Universitätsmedizin Berlin, Hindenburgdamm 30,

12200 Berlin, Germany

e-mail: ulf.gast@charite.de
Methods We examined short- $(n=50)$, middle- $(n=19)$ and long-distance $(n=109)$ athletes at the 15th European Masters Championships in Poznań, Poland. Dual X-ray absorptiometry was used to measure areal bone mineral density (aBMD) and lean tissue mass. Maximal countermovement jump, multiple one-leg hopping and maximal grip force tests were performed. Results Short-distance athletes showed significantly higher aBMD at the legs, hip, lumbar spine and trunk compared to long-distance athletes $(p \leq 0.0012)$. Countermovement jump performance, hop force, grip force, leg lean mass and arm lean mass were greater in short-distance athletes $(p \leq 0.027)$. A similar pattern was seen in middle-distance athletes who typically showed higher aBMD and better neuromuscular performance than long-distance athletes, but lower in magnitude than short-distance athletes. In all athletes, aBMD was the same or higher than the expected age-adjusted population mean at the lumbar spine, hip and whole body. This effect was greater in the short- and middle-distance athletes.

Conclusions The stepwise relation between short-, middle- and long-distance athletes on bone suggests that the higher-impact loading protocols in short-distance disciplines are more effective in promoting aBMD. The regional effect on bone, with the differences between the groups being most marked at loadbearing regions (legs, hip, spine and trunk) rather than nonload-bearing regions, is further evidence in support of the idea that bone adaptation to exercise is dependent upon the local loading environment, rather than as part of a systemic effect.

Keywords Bone $\cdot$ Elderly $\cdot$ Exercise $\cdot$ Mechanical loading $\cdot$ Muscle $\cdot$ Osteporosis

\section{Introduction}

With an ageing population in Western countries, age-related diseases and decline, such as osteoporosis, sarcopenia and loss of neuromuscular performance, will in the future result 
in a greater burden for health care systems. For the management and prevention of these diseases, it is important to understand if physical activity can influence these parameters, and if so, then what kind of physical activity is most effective.

Elite level Masters athletes represent an ideal population to examine the relationship between different types of physical activity and aspects of musculoskeletal form and function, such as bone mineral density, muscle bulk and neuromuscular performance $[1,2]$. Since participants in track and field Masters championships have been participating in their chosen sport for a number of years, their body has adapted to the loads placed upon it. Athletes participating in short- (up to $400 \mathrm{~m}$ sprint, triple jump and long jump), middle- $(800$ to $1,500 \mathrm{~m})$ and long-distance $(2,000 \mathrm{~m}$ to marathon) disciplines enable the examination of loading patterns ranging from explosive, high-load (short-distance disciplines) loads to weight-bearing, repetitive (long-distance disciplines) loads.

Evidence suggests that high-load and high rate of loading (e.g. jumping, sprinting) regimes provide the greatest stimulus for bone formation. This is supported by experimental data from animal studies $[3,4]$, by cross-sectional studies in humans $[5,6]$. A greater extent of bone deformation and higher rate of bone deformation occur in high-impact activities such as sprinting [7]. There have been few studies that have examined different sports types in older elite athletes, and one study [8] found short-distance (sprint) athletes to have greater tibia bone mass and cortical area than longdistance runners, and another [9] found higher areal bone density in sprint athletes. Similarly, explosive type training has been shown to improve muscular power [10].

The aim of the current study was to examine short-, middle- and long-distance disciplines in elite Masters track athletes in relation to bone mineral density, neuromuscular performance and lean mass. Based on data available to date $[8,9,11,12]$, we hypothesized that short-distance athletes would show a higher bone mineral density, explosive power during countermovement jump testing and higher lean mass than long-distance athletes. A secondary hypothesis was that the rate of age-related decline in bone mineral density would be slower in short- than long-distance athletes.

\section{Materials and methods}

\section{Subject recruitment}

This study was conducted at the 15th European Masters Championships in Poznań, Poland from 19 to 30 July 2006. The ethics committee of the Karol Marcinkowski Medical University in Poznań approved the study. Subjects were recruited from the athletes attending the championship and gave their informed written consent. Testing was performed locally at the sport facility in Poznań. Due to differences in bone density in individuals of African or Asian origin [13], only Caucasian individuals were included in the current study. Subjects' self-rated best discipline resulted in their assignment to the short-distance (sprinters up to $400 \mathrm{~m}$ distance as well as long and triple jumpers), middle-distance ( 800 to $1,500 \mathrm{~m}$ runners) or long-distance (runners $2,000 \mathrm{~m}$ and above) groups. Other self-rated best disciplines or subjects unable to give a single self-rated best discipline were excluded from the study. Athletes who were pregnant or had a current injury were excluded from the study.

The European Masters Championships were organized such that all individuals who met the minimum age requirements were allowed to participate. Hence, there could have potentially been athletes who cite a particular discipline as their best, but not necessarily have trained intensely for a number of years. Thus, to include those subjects who would show the greatest neuromusculoskeletal adaptation to their chosen discipline, we aimed to recruit the "best of the best" by approaching athletes who made it to the final and then completed this final race.

After assessing the inclusion criteria, subjects were questioned regarding date of birth, duration of sport participation and duration of training per week. Female subjects were also questioned regarding age of menarche and their age at menopause if this had occurred. Subject height and body mass were measured with a Seca 764 device (Vogel \& Halke $\mathrm{GmbH}$, Hamburg, Germany). Then, after initial screening, bone density, body composition, countermovement jump, multiple one-leg hopping and grip force testing were performed. The characteristics of the subject collective are presented in Table 1.

Table 1 Subject characteristics

\begin{tabular}{llll}
\hline Parameter & \multicolumn{2}{l}{ Distance } & \\
\cline { 2 - 3 } & Short & Middle & Long \\
\hline Number of males & 32 & 11 & 65 \\
Number of females & 18 & 8 & 44 \\
Age (years) & $53.6(14.4)$ & $52.6(11.0)$ & $57.5(11.2)$ \\
Age range (years) & $35-94$ & $38-75$ & $35-78$ \\
Height (cm) & $171.7(7.9)$ & $169.0(7.8)$ & $168.2(8.4)$ \\
Body mass (kg) & $68.3(10.2)$ & $60.2(6.3)$ & $62.8(8.2)$ \\
Female age at menarche & $12.6(1.3)$ & $14.8(1.5)$ & $14.3(1.7)$ \\
Career duration (years) & $13.6(12.8)$ & $14.4(12.9)$ & $16.4(12.6)$ \\
Career duration & $1.5-80.7$ & $2.4-47.1$ & $1.5-52.7$ \\
$\quad$ range (years) & & & \\
Training hours per week & $8.0(4.0)$ & $7.7(4.5)$ & $8.8(4.3)$ \\
\hline
\end{tabular}

Height ( $p=0.047$ ) and body mass $(p=0.0003)$ differed between groups (otherwise $p \geq 0.078$ ). The proportion of female and male athletes did not differ between groups $\left(\chi^{2}=0.15, p=0.93\right)$. 
Dual X-ray absorptiometry: lumbar spine, total hip and whole body scanning

Areal bone mineral density (aBMD in grams per square centimeter) of the lumbar spine (L1-L4 in anterioposterior projection), the proximal femur (total) and femoral neck on the left and right sides as well as whole body were measured using dual X-ray absorptiometry (DXA) with a Lunar Prodigy Advance (General Electric Company, Waukesha, Wisconsin, USA). Scans were performed according to the standard manufacturer's protocol. T-scores, which express the difference to young adults of the same gender with peak bone mass, as well as Z-scores, which express the difference to the sex- and ageadjusted population mean, for each of these regions were also calculated using the reference population included in the manufacturer software. aBMD in the legs, arms and trunk sub-regions was derived from the whole body scan. Whole body, arm, trunk and leg lean mass (in kilograms) were also calculated from whole-body scans. All scanning and analyses were performed by the same operator to ensure consistency and followed standard quality control procedures. Where left and right sides were scanned (arms, legs, proximal femur), these values were averaged between the left and right sides of the body prior to further analysis.

Data from our own research group (unpublished observations) show a coefficient of variation and Pearson's correlation co-efficient of, respectively, $0.81 \%$ and 0.996 for total hip aBMD when averaged between left and right sides (two repeated measurements after immediate repositioning; $n=64)$ and $1.31 \%$ and 0.982 for scans of the lumbar spine (two repeated measurements after immediate repositioning; $n=25)$. Based upon the subject numbers of the current cross-sectional study, and assuming a power of 0.8 and an alpha level of 0.05 , an effect size of $5.7 \%$ for hip aBMD, and $4.4 \%$ for lumbar spine aBMD should be able to be detected for the short-distance versus long-distance comparison and, respectively, 9.1 and $6.9 \%$ for the short-distance versus middle-distance comparison. These effect size calculations were performed with $G^{*}$ Power version 3.1.2 [14].

\section{Neuromuscular performance}

Countermovement jump testing was performed on a ground reaction force platform (Leonardo Mechanograph GRFP, Novotec GmbH, Pforzheim, Germany). Subjects first stood on the $60 \times 60-\mathrm{cm}$ testing platform with their arms resting at their sides. Before the first jump, the subject's body mass was measured for use in subsequent calculations. Subjects were then instructed to perform a countermovement (i.e. a brief squat beforehand) jump as high as possible. The same verbal encouragement was given for every jump by the same operator. Three jumps were performed during each testing session with a break of $1 \mathrm{~min}$ between each jump. The peak force developed for the ascending part of the jump was calculated along with the peak power (product of force and velocity) and then expressed relative to body mass. Maximum jump height was also calculated based on the numeric integration of vertical velocity data. The "Esslinger Fitness Index" was calculated as a Z-score of jump power per unit body mass relative to an age- and gendermatched reference population $[15,16]$. Software provided by the manufacturer (Leonardo Mechanography v4.2) was used for recording and storage of data and for subsequent calculation of the variables of interest. The data from the jump of maximal jump height from all three trials were used in further analysis. The short-term co-efficient of variation in adult and elderly subjects for peak jump power as a function of body mass is $3.6 \%$ [17].

For multiple one-leg hopping, the same testing apparatus was used as for the countermovement jump. In the course of ten continuous single leg hops, the subject was required to maintain their knee close to full extension [18], and no arm swing was permitted. Due to the repeated concentric-eccentric cycles of straight-knee hopping, hence with storage of elastic energy in the musculotendinous system, it was possible to examine peak force development at the ankle joint [19]. Subjects were instructed to maintain the heel off the ground for all hops. Verbal encouragement was given for every test. Each leg was tested twice with a break of 1 min between test runs. Using the same software as per the countermovement jump test, peak hop force was calculated and expressed relative to body mass. Data from the single hop of greatest force from the two sets of ten consecutive hops on each leg were chosen for further analysis.

Maximal grip force testing was performed in standing using a digital hand dynamometer (Takei Scientific Instruments Co. Ltd, Tokyo, Japan). The shoulder was placed in an adducted and neutrally rotated position with the elbow in full extension [20]. Three repetitions were performed on the subject's dominant hand with a 30 -s break between tests. The peak value (in Newtons) was used in further analysis.

\section{Statistical analysis}

Linear mixed-effects models [21] were used to assess each parameter. A main effect of athlete group (short, middle and long distance) with linear covariates of age, height, body mass and duration of training was included in the models. Gender and a gender $\times$ group interaction were included in each model. Allowances for heterogeneity of variance due to athlete group were made where necessary. Analysis of co-variance (ANCOVA) was then performed for each model. Nonsignificant covariates were removed from models before the adjusted values were calculated. Subsequently, a priori comparisons were conducted comparing the middle- and longdistance groups to the short-distance group as well as the middle-distance group to the long-distance group. Differences 
between genders were expected for a number of the variables evaluated. The focus of the current work was, however, on the differences between athlete groups whilst controlling for differences due to gender. If a significant gender $\times$ group interaction was found, further models were conducted to compare between genders for each athlete group.

To assess the relationship between age and any impact of athlete group, linear regression of age and the outcome variable was examined before and after controlling for the influence of other covariates (height, body mass, training duration), and additional models were constructed including an age $\times$ group interaction. An $\alpha$ of 0.05 was taken for statistical significance. The " $R$ " statistical environment (version 2.10.1, www.r-project.org) was used for all analyses.

Unless otherwise stated, all values are reported as mean (SD). Reported absolute values have been adjusted for subject age, height, body mass and training duration. Results of analyses were similar if the sample was restricted to those athletes who had trained between 5 and 47.1 years (i.e. by ensuring a similar training duration in each group).

Partial correlation (Pearson's) analyses were also performed on whole body aBMD, lumbar aBMD, total hip aBMD, leg aBMD, lean mass legs, peak jump power, hop force and grip force. The influence of subject age, height, body mass, gender and training duration was controlled. To examine the role that sport-type played on the correlations, additional analyses were performed also controlling for athlete group. A Bonferroni adjustment was performed for these correlation analyses.

\section{Results}

The subject characteristics and training history are presented in Table 1. Of the female subjects, 28 had reached menopause [short distance, $n=3$; ages, 53,53 and 58 years; middle distance, $n=2$, ages 43 and 48 years, long distance, $n=23$, mean (SD) age, 50.6 (4.4)years]. The proportion of post-menopausal women did not differ significantly between groups $\left(\chi^{2}=3.5, p=0.17\right)$ and randomly excluding post-menopausal females in the mid- and long-distance groups to ensure similar proportions did not influence the results (data not shown).

\section{Bone}

After controlling for anthropometric variables and training duration, significant differences between groups were seen on ANCOVA for total hip $(p=0.0011)$, femoral neck ( $p=$ $0.0033)$, lumbar spine $(p<0.0001)$, whole-body total $(p=$ $0.0011)$, trunk $(p<0.0001)$ and leg $(p<0.0001)$ aBMD. The short-distance athletes had significantly higher aBMD in these body regions than the long-distance athletes (Table 2 and Fig. 1 top section). A similar pattern was apparent between short- and middle-distance athletes, although the differences between these two groups only reach significance at the lumbar spine. Middle-distance athletes typically had higher aBMD than the long-distance athletes, but this only reached statistical significance for whole-body aBMD and also leg aBMD. No significant differences between groups were seen for arm aBMD values $(p=0.16)$.

T-score data from total hip $(p=0.0003)$, femoral neck ( $p=$ $0.0024)$, lumbar spine $(p<0.0001)$ and whole-body $(p=$ 0.0003 ) scans showed significant differences between groups (Fig. 2). The short-distance athletes showed aBMD levels above their expected sex-adjusted peak bone mass mean at all scan regions $(p \leq 0.037)$, whereas the middledistance athletes showed this only for whole-body scans ( $p=$ 0.005 ) and the long-distance athletes were below their expected sex-adjusted peak bone mass mean for lumbar spine $(p<0.0001)$ and total hip $(p=0.00001)$ with no significant difference compared to the general population for whole-body scans. The Z-scores showed a similar pattern; however, all the athlete groups remained above their expected age- and sex-adjusted mean (Fig. 2).

In some cases, a significant gender $\times$ group effect was seen on ANCOVA for data from whole-body scanning: total aBMD $(p=0.025)$, arm aBMD $(p=0.008)$, trunk aBMD $(p=0.042)$ and T-score $(p=0.028)$. These effects were driven by female long-distance athletes showing lower $[-7.4(14.0) \% ; p=$ $0.0001]$ whole-body total aBMD than their male counterparts. Male athletes showed higher arm aBMD than females, but this effect was stronger in the middle- [males $+20.4(10.3) \%$ versus females; $p<0.0001]$ and long-distance $[+15.9(18.6) \% ; p<$ $0.0001]$ athletes than in short-distance $[+6.8(17.2) \% ; p=$ 0.04]. At the trunk, no significant male-female differences were seen on direct comparisons within groups, but male athlete long-distance trunk aBMD tended to be higher than their female counterparts $[+3.6(15.1) \%, p=0.068]$, with male short-distance trunk aBMD marginally lower than the female short-distance athletes $[-3.6(15.4) \% ; p=0.17]$. In the shortdistance group, male athletes showed significantly $(p=$ 0.0045) lower T-scores than their female counterparts, with no significant differences in the other athlete groups.

Lean mass, countermovement jump, multiple one-leg hopping and grip force

For all lean mass $(p \leq 0.036)$ variables, there was evidence on ANCOVA for significant differences between the athlete groups. Short-distance athletes showed greater lean mass than the long-distance athletes, but this only reached significance in the arms, legs and whole body (Table 2 and Fig. 1 middle section) and no significant differences compared to middle-distance athletes. Middle-distance athletes showed a similar pattern with significantly greater trunk and wholebody lean mass than long-distance athletes. 
Table 2 Bone mineral density, lean mass and neuromuscular performance
ANCOVA showed the differences between the groups to be significant for all variables $(p \leq 0.036)$ except arm bone density $(p=0.16)$. See "Results" section for more details on differences in arm bone mineral density between genders

$* p<0.05 ; * * p<0.01$;

$* * * p<0.001$, and refer to the difference to the short-distance group. $* * * * p<0.05$;

$* * * * * p<0.01 ; * * * * * * p<0.001$, and refer to the difference between the middle- and longdistance groups. g: $9.81 \mathrm{~m} / \mathrm{s}^{2}$

\begin{tabular}{llll}
\hline Parameter & Distance & & \\
\cline { 2 - 4 } & Short & Middle & Long \\
\hline Bone mineral density $\left(\mathrm{g} / \mathrm{cm}^{2}\right)$ & & & \\
Lumbar spine (L1-L4) & $1.27(0.19)$ & $1.16(0.16)^{*}$ & $1.11(0.16)^{* * *}$ \\
Total hip & $1.09(0.13)$ & $1.05(0.10)$ & $1.01(0.13)^{* * *}$ \\
Femoral neck & $1.03(0.14)$ & $0.98(0.08)^{*}$ & $0.95(0.12)^{* * *}$ \\
Whole body total & $1.24(0.10)$ & $1.24(0.08)^{* * * *}$ & $1.19(0.09)^{* *}$ \\
Arms & $1.02(0.10)$ & $1.05(0.10)$ & $1.00(0.12)$ \\
Trunk & $0.96(0.08)$ & $0.93(0.08)$ & $0.90(0.07)^{* * *}$ \\
Legs & $1.44(0.13)$ & $1.42(0.10)^{* * * * *}$ & $1.34(0.13)^{* * *}$ \\
Lean mass (kg) & & & $5.48(0.76)^{*}$ \\
Arms & $5.79(0.73)$ & $5.78(0.74)$ & $24.80(2.26)$ \\
Trunk & $25.33(2.13)$ & $25.80(1.50)^{* * * *}$ & $16.94(1.57)^{* * *}$ \\
Legs & $17.84(1.50)$ & $17.40(1.26)$ & $50.83(4.51)^{*}$ \\
Whole body total & $52.44(3.98)$ & $52.73(3.29)^{* * * * *}$ & \\
Neuromuscular tests & & & $36.55(6.12)^{* * *}$ \\
Peak jump power/body mass (W/kg) & $48.24(8.03)$ & $42.90(6.13)^{* *}, * * * * * *$ & $2.34(0.38)^{* *}$ \\
Peak jump force/body mass (g) & $2.52(0.33)$ & $2.48(0.36)$ & $0.32(0.06)^{* * *}$ \\
Jump height (m) & $0.42(0.06)$ & $0.38(0.06)^{* * * * * * * * *}$ & $3.26(0.36)^{* * *}$ \\
Peak hop force/body mass $(\mathrm{g})$ & $3.57(0.35)$ & $3.42(0.30)^{* * * *}$ & $360(59)^{*}$ \\
Maximal grip force (Newton) & $387(69)$ & $386(68)$ & \\
\hline
\end{tabular}

Countermovement jump $(p \leq 0.0085)$ and grip force $(p=$ 0.029 ) variables also showed significant differences between the athlete groups on ANCOVA. In relation to functional tests, short-distance athletes showed greater peak jump power per unit body mass, peak force per unit body mass, jumping height, hop force per unit body mass and grip force than long-distance athletes (Table 2 and Fig. 1 bottom section). Compared to middle-distance athletes, short-distance athletes showed only significantly greater peak jump power per unit body mass. Middle-distance athletes also performed better at the countermovement jump, multiple one-leg hop and grip strength tests, but the differences between the two groups were only significant for peak jump power per unit body mass, jumping height and peak hop force.

In relation to the lean mass and functional variables, a significant gender $\times$ group interaction was seen for jump height only $(p=0.0035)$. Whilst males showed a greater jumping height than females in general, this effect was stronger for short-distance $[+28(19) \%$ greater jump height for male versus female athletes; $p<0.0001]$ than for middle$[+21(20) \% ; p=0.016]$ and long-distance $[+16(30) \%$; $p=0.0004]$ athletes.

The Z-scores for peak countermovement jump power per unit body mass differed between groups $(p<0.0001)$. Short$(p<0.0001)$ and middle-distance $(p=0.015)$ athletes were significantly above the average of the reference population, whereas the long-distance athletes $(p=0.0007)$ were worse than the average (Fig. 3).

\section{Relationship to age}

All aBMD variables showed a negative relationship with age, but when controlling for the effects of subject height, body mass and training duration, the statistical significance of this relationship persisted only for total hip aBMD, whole-body total aBMD and leg aBMD. No significant differences were seen between groups on the relationship between age and the aBMD variables $(p \geq 0.51)$.

With the exception of arm lean mass, the remaining lean mass and all functional parameters declined with increasing athlete age. These effects were, after controlling for co-variates, statistically significant for all parameters. There were no differences between groups in the linear rate of decline of these parameters with age $(p \geq 0.13)$.

\section{Correlation analyses}

Correlations between aBMD variables were moderate to high and persisted once the effect of athlete group was controlled (Table 3). This was similarly the case for correlations between neuromuscular function tests. Weak to moderate partial correlations were seen between leg aBMD and peak jump power per unit body mass as well as peak hop force. However, these correlations were weaker and no longer significant once the effect of athlete group was controlled. 
Fig. 1 Differences compared to short-distance athletes of bone density (top), lean mass (middle) and neuromuscular function $(\text { bottom })^{*} p<0.05$; ${ }^{\dagger} p<0.01 ;{ }^{\ddagger} p<0.001$ and refer to the difference to the shortdistance group. $a p<0.05$; $b p<0.01 ; c p<0.001$ and refer to the difference between the middle- and long-distance groups. See "Results" section for more details on differences in arm bone mineral density between genders middle-distance $\mathbf{a}$ long-distance
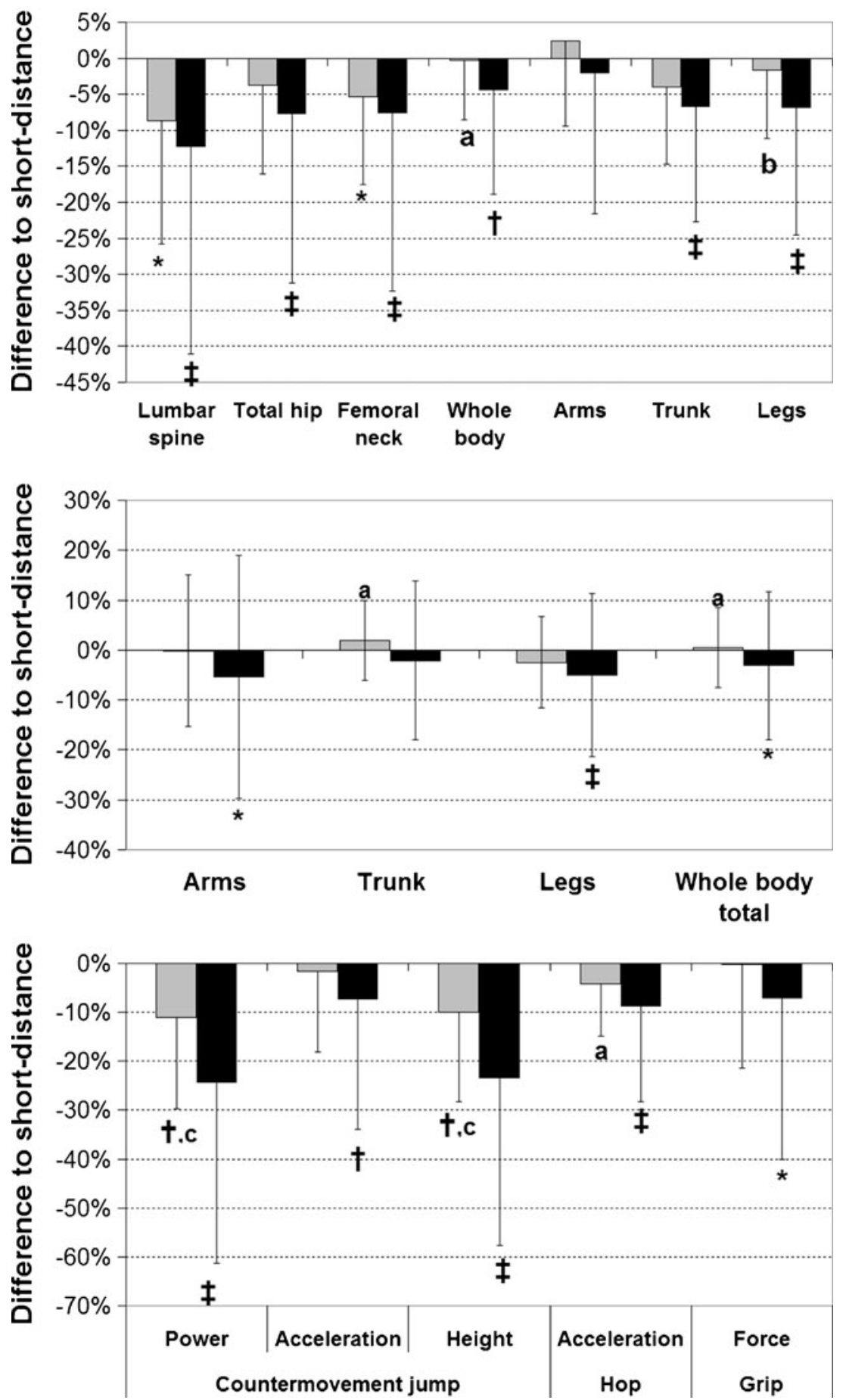

\section{Discussion}

The current study examined elite Master track athletes and found, after adjusting for age, height, gender and training duration, that the short-distance athletes (sprinters up to $400 \mathrm{~m}$ distance as well as long and triple jumpers) had a higher bone density in all body regions, with the exception of the arms, than long-distance athletes. Countermovement jump performance, peak hop force and grip force were greater in the short-distance athletes than long-distance athletes, and these athletes also showed greater lean mass in the arms and legs than long-distance athletes. A similar pattern was observed for comparisons between short- and middle-distance athletes, but these effects were smaller in magnitude and 
Fig. 2 Differences to peak bone density (T-score) and expected age-adjusted bone density (Z-score) from dual $\mathrm{X}$-ray absorptiometry. ${ }^{\#} p<0.05$; ${ }_{p<0.01 ;} \|_{p<0.001 \text { refer to the }}$ difference to zero. $a p<0.05$ refers to the difference between the middle- and long-distance groups. ${ }^{*} p<0.05 ;{ }^{\dagger} p<0.01$; ${ }^{{ }^{1}} p<0.001$ and refer to the difference of the middle- or longdistance groups to the short-distance group
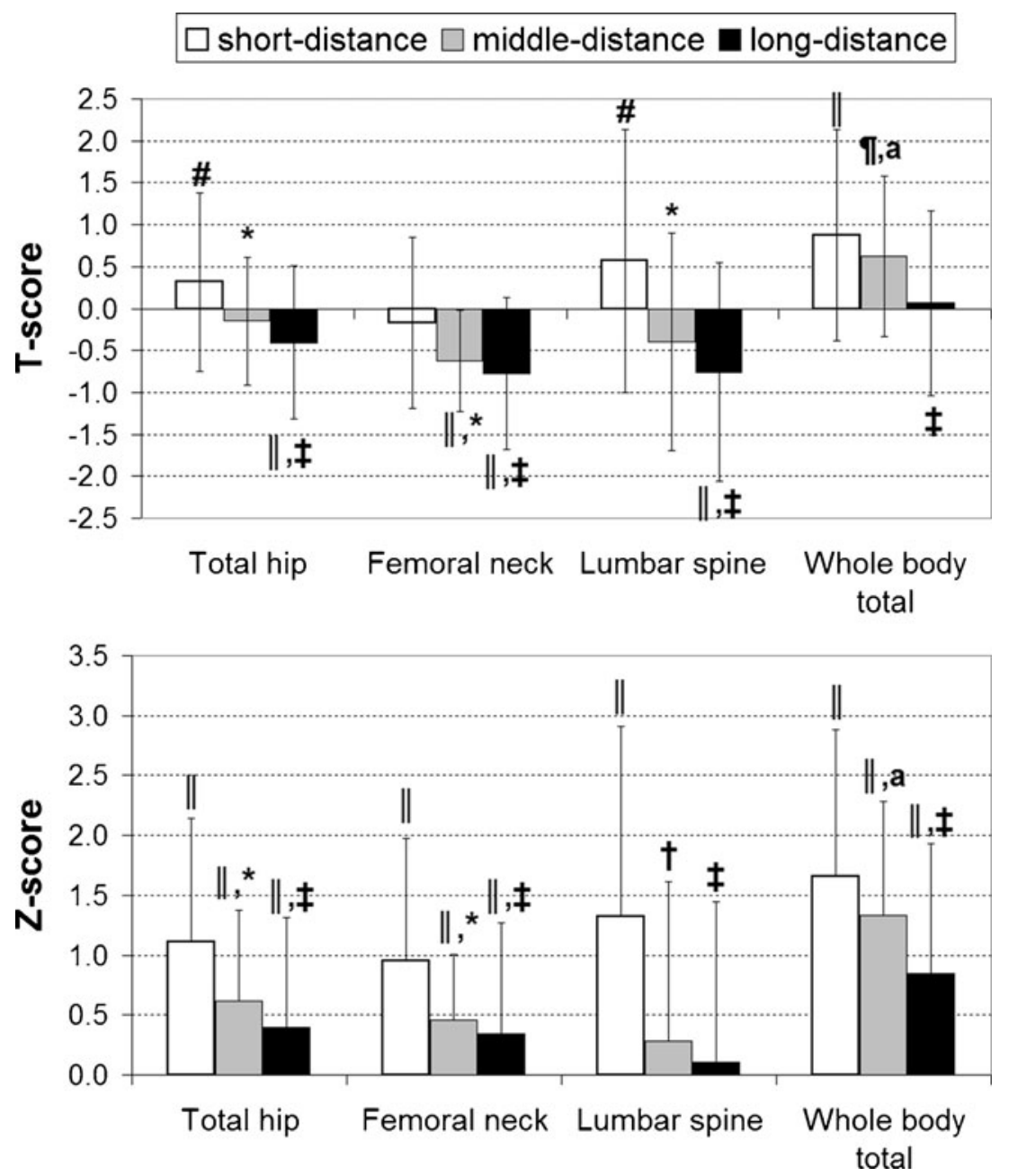

significant for bone density at the lumbar spine and femoral neck, T-scores at the lumbar spine, total hip and femoral neck and countermovement jump power and height.

These findings support the primary hypothesis that participation of older individuals in short-distance track sports, which involve high-load and high-rate of loading impact exercise, shows greater bone density and explosive power. The stepwise effect on bone, lean mass and neuromuscular performance from short-, middle- to long-distance athletes also gives weight to the argument that the loading protocols as part of these disciplines are responsible for the findings in the outcome parameters. Also, the effect on bone was
Fig. 3 Peak jump power expressed as age- and genderadjusted Z-scores. $\|_{p<0.001}$ refers to the difference to zero. $c p<0.001$ refers to the difference between the middle- and long-distance groups. ${ }^{*} p<0.001$ refers to the difference of the middle- or long-distance groups to the short-distance group

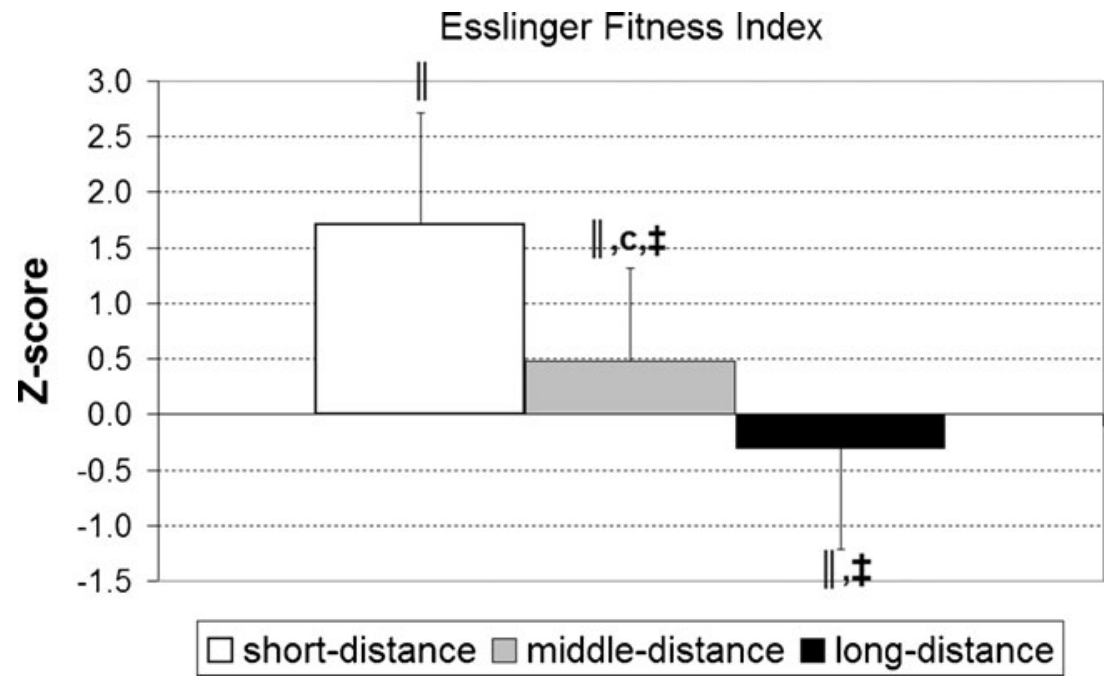


regional with the differences between the groups being most marked at load-bearing regions (legs, hip, spine and trunk) rather than non-load-bearing regions (arms). This is further evidence in support of the idea that bone adaptation is dependent upon the local loading environment, rather than as part of a systemic effect $[22,23]$. Basic science in the study of bone has indeed shown that high loads and rates of loading provide a stronger stimulus for bone formation [3, 4]. Similar cross-sectional studies in younger individuals [5, $6,24]$ have also shown a graded effect on bone when progressing from high-impact exercise types (e.g. sprinting, jumping, boxing), to high-load but low-impact (e.g. resistive exercise) and then low-impact repetitive exercise (e.g. jogging) and non-impact repetitive exercise (e.g. swimming). The gold standard for exercise interventions is, however, a randomized controlled trial. Whilst there are some prospective human studies [25] supporting the ideas obtained from animal research [3, 4] and cross-sectional studies [5, 6], more randomized controlled trials are needed. Also, in the prospective trials to date, a number of studies to date incorporate high-impact exercise mixed with other exercise forms which makes it difficult to assess the impact of specific loading type [26]. Whilst the findings of the current and prior studies support the hypothesis that high-impact and explosive exercise can have a more positive effect on bone, lean mass and maximal neuromuscular performance than other exercise forms in older individuals, further prospective controlled trials need to be performed to test this hypothesis.

In terms of the secondary goal of the study, whilst we did consistently see a negative relationship (decline) in the outcome parameters with age, we did not find a difference between sport types in this decline. These results from the current cross-sectional study suggest that participation in short-distance sport types does not slow the "rate of decline" with age per se. Short-distance explosive sport types nonetheless appear more beneficial in that they result in a higher absolute value of each given parameter at a given age. The findings that the kind of exercise modality does not impact on the rate of age-related decline would need to be further examined as part of longitudinal studies.

The T- and Z-scores from DXA scanning and Z-scores from countermovement jump testing provide a unique insight into the impact of different sport types compared to the general population. These scores are generated compared to a reference population included with the software and give an indication of an individual's bone mineral density compared to their sex-matched young reference of peak bone mass (T-score), their expected age-adjusted value (Z-score) and their age-expected peak jump power (Esslinger Fitness Index Z-score). Since the long-distance group performs large volumes of weight-bearing physical activity, one might expect that they would show at least a slightly better bone density than the general population. This is indeed the case as shown by the Z-scores at hip and whole body but not lumbar spine. However, the T-scores imply that the longdistance athletes have lost more bone since their youthful peak bone density than athletes of other disciplines. Also, these athletes were below the average of the reference population for countermovement jump performance. In contrast, the short-distance athletes showed higher bone mineral density than might have been expected at peak bone density (as shown by T-scores) or for individuals of same age and sex (as shown by Z-scores) as well as countermovement jump performance. A similar pattern was seen for middle-distance athletes, but the effect was not as strong as in the shortdistance group. These data provide further support to the idea that weight-bearing physical activity is important for retaining bone with age (data from long-distance group), but that explosive, high-impact, loading types are more effective in achieving this goal (results from short-distance group).

The correlation analyses were performed to attempt to gain a deeper insight into the relationship between sport

Table 3 Partial correlation coefficients between bone, leg lean mass, jump power, hop force and grip force

\begin{tabular}{|c|c|c|c|c|c|c|c|c|}
\hline Parameter & $\begin{array}{l}\text { Whole body } \\
\text { BMD }\end{array}$ & $\begin{array}{l}\text { Lumbar } \\
\text { BMD }\end{array}$ & $\begin{array}{l}\text { Total hip } \\
\text { BMD }\end{array}$ & $\begin{array}{l}\text { Leg } \\
\text { BMD }\end{array}$ & Lean mass legs & $\begin{array}{l}\text { Peak jump } \\
\text { power }\end{array}$ & $\begin{array}{l}\text { Peak hop } \\
\text { force }\end{array}$ & $\begin{array}{l}\text { Grip } \\
\text { force }\end{array}$ \\
\hline Whole body BMD & & $0.77 * *$ & $0.78 * *$ & $0.86^{* *}$ & 0.09 & 0.15 & 0.17 & 0.06 \\
\hline Lumbar BMD & $0.75^{* *}$ & & $0.65^{* *}$ & $0.59 * *$ & 0.01 & 0.19 & 0.15 & 0.00 \\
\hline Total hip BMD & $0.77 * *$ & $0.61 * *$ & & $0.80 * *$ & 0.12 & 0.16 & 0.25 & 0.13 \\
\hline Leg BMD & $0.85^{* *}$ & $0.52 * *$ & $0.79 * *$ & & 0.20 & 0.24 & $0.26^{*}$ & 0.15 \\
\hline Lean mass legs & -0.04 & -0.18 & -0.01 & 0.03 & & $0.50^{* *}$ & $0.41^{* *}$ & 0.24 \\
\hline Peak jump power & -0.06 & -0.09 & -0.06 & -0.06 & $0.29 *$ & & $0.59^{* *}$ & 0.23 \\
\hline Peak hop force & 0.05 & -0.01 & 0.15 & 0.10 & 0.25 & $0.42 * *$ & & 0.23 \\
\hline Grip force & -0.01 & -0.10 & 0.06 & 0.05 & 0.15 & 0.07 & 0.14 & \\
\hline
\end{tabular}

Values are Pearson's partial correlation coefficient controlling for subject age, height, mass, gender and training duration (top right triangle in grey). In the bottom left triangle, the effect of athlete group has also been controlled

${ }^{*} p<0.05$ and ${ }^{* *} p<0.001$ after Bonferroni correction 
type and select key outcome variables. After controlling for the effects of subject age, height, mass and gender, significant weak to moderate correlations were seen between leg aBMD and measures of leg lean mass, peak jump power per unit body mass and peak hop force. However, these effects disappeared once the athlete group was controlled. This implies that whilst there is some relationship between aBMD and neuromuscular performance, the the kind of sport or loading pattern influences both of them independently.

It is important to mention some of the limitations of the current study. The current study utilized a cross-sectional design. Consequently, whilst the results provide support for certain hypotheses, further prospective trials will need to be performed to examine these issues further. Also, the current study could not be strictly randomized: we approached athletes who participated in the final race as we thought these individuals would have the greatest adaptation to their chosen sport. Related to this, there could be a kind of "self-selection bias" [6] where individuals who naturally have greater explosive power may more likely to be drawn to a particular sport type. This may well be true, and similarly, this issue can only be avoided by implementing subsequent prospective studies. We also do not have information on physical activity profiles of our subjects during childhood and adolescence and do not have specific details on the training programs undertaken by the athletes beyond training duration and frequency. Furthermore, the current study did not include a control group of sedentary individuals, which limits the interpretation of some parameters where $\mathrm{T}$ - and Z-scores were not available. Finally, as shown by the sensitivity analysis, in the middle-distance group the smaller number of subjects may have been responsible for the non-significance for the comparison between groups for some parameters.

In conclusion, the main findings were that short-distance athletes showed significantly higher bone density at the legs, hip, lumbar spine and trunk compared to long-distance athletes. Countermovement jump performance, multiple one-leg hopping and grip force were also greater in short-distance athletes as well as leg and arm lean mass. A similar pattern was seen with middle-distance athletes who typically showed higher bone mineral density and better neuromuscular performance than long-distance athletes, but less so than short-distance athletes. The stepwise effect between short-, middle- and long-distance athletes on bone suggests that the higher-impact loading protocols in middle- and short-distance disciplines are more effective in promoting bone mineral density. Also, the regional effect on bone in these ambulant sport types, with the differences between the groups being most marked at loadbearing regions (legs, hip, spine, trunk) rather than non-loadbearing regions (arms), is further evidence in support of the idea that bone adaptation to exercise is dependent upon the local loading environment, rather than as part of a systemic effect.
Acknowledgments The authors wish to thank the subjects involved in the study for donating their time. Tilo Blenk, Arndt Boshof, Constanze Gutwasser, Biljana Radonic and Frank Touby of the Center of Muscle and Bone Research are thanked for their assistance in the course of the project. Our colleagues in Poland and Manchester are thanked for the enjoyable collaboration and assistance in the course of the data collection.

Conflicts of interest Rainer Rawer is an employee of Novotec Medical. Dieter Felsenberg acts as an unpaid consultant to Novotec Medical for the exploitation of the study's results. All other authors have no conflict of interest.

Grants Institutional funding only was used for the implementation of this study.

\section{Reference}

1. Rittweger J, Kwiet A, Felsenberg D (2004) Physical performance in aging elite athletes - challenging the limits of physiology. J Musculoskelet Neuronal Interact 4:159-160

2. Lazarus NR, Harridge SD (2007) Inherent ageing in humans: the case for studying master athletes. Scand J Med Sci Sports 17:461-463

3. Srinivasan S, Ausk BJ, Poliachik SL et al (2007) Rest-inserted loading rapidly amplifies the response of bone to small increases in strain and load cycles. J Appl Physiol 102:1945-1952

4. O'Connor JA, Lanyon LE, MacFie H (1982) The influence of strain rate on adaptive bone remodelling. J Biomech 15:767-781

5. Nikander R, Sievänen H, Heinonen A et al (2005) Femoral neck structure in adult female athletes subjected to different loading modalities. J Bone Miner Res 20:520-528

6. Nikander R, Sievänen H, Uusi-Rasi K et al (2006) Loading modalities and bone structures at nonweight-bearing upper extremity and weight-bearing lower extremity: a pQCT study of adult female athletes. Bone 39:886-894

7. Burr DB, Milgrom C, Fyhrie D et al (1996) In vivo measurement of human tibial strains during vigorous activity. Bone 18:405-410

8. Wilks DC, Winwood K, Gilliver SF et al (2009) Bone mass and geometry of the tibia and the radius of master sprinters, middle and long distance runners, race-walkers and sedentary control participants: a pQCT study. Bone 45:91-97

9. Nowak A, Straburzynska-Lupa A, Kusy K et al (2010) Bone mineral density and bone turnover in male masters athletes aged 40-64. Aging Male 13:133-141

10. Paavolainen L, Häkkinen K, Hämäläinen I et al (1999) Explosivestrength training improves $5-\mathrm{km}$ running time by improving running economy and muscle power. J Appl Physiol 86:1527-1533

11. Wilks DC, Winwood K, Gilliver SF et al (2009) Age-dependency in bone mass and geometry: a pQCT study on male and female master sprinters, middle and long distance runners, race-walkers and sedentary people. J Musculoskelet Neuronal Interact 9:236246

12. Michaelis I, Kwiet A, Gast U et al (2008) Decline of specific peak jumping power with age in master runners. J Musculoskelet Neuronal Interact 8:64-70

13. Bachrach LK, Hastie T, Wang MC et al (1999) Bone mineral acquisition in healthy Asian, Hispanic, black, and Caucasian youth: a longitudinal study. J Clin Endocrinol Metab 84:47024712

14. Faul F, Erdfelder E, Lang A-G et al (2007) G*Power 3: a flexible statistical power analysis for the social, behavioral, and biomedical sciences. Behav Res Methods 39:175-191 
15. Fricke O, Weidler J, Tutlewski B et al (2006) Mechanography-a new device for the assessment of muscle function in pediatrics. Pediatr Res 59:46-49

16. Runge M, Rittweger J, Russo CR et al (2004) Is muscle power output a key factor in the age-related decline in physical performance? A comparison of muscle cross section, chair-rising test and jumping power. Clin Physiol Funct Imaging 24:335-340

17. Rittweger J, Schiessl H, Felsenberg D et al (2004) Reproducibility of the jumping mechanography as a test of mechanical power output in physically competent adult and elderly subjects. J Am Geriatr Soc 52:128-131

18. Farley CT, Morgenroth DC (1999) Leg stiffness primarily depends on ankle stiffness during human hopping. J Biomech 32:267-273

19. van Ingen Schenau GJ, Bobbert MF, Huijing PA et al (1985) The instantaneous torque-angular velocity relation in plantar flexion during jumping. Med Sci Sports Exerc 17:422-426

20. Incel NA, Ceceli E, Durukan PB et al (2002) Grip strength: effect of hand dominance. Singapore Med J 43:234-237
21. Pinheiro JC, Bates DM (2000) Mixed-effects models in S and S-PLUS. Springer, Berlin

22. Sugiyama T, Price JS, Lanyon LE (2010) Functional adaptation to mechanical loading in both cortical and cancellous bone is controlled locally and is confined to the loaded bones. Bone 46:314321

23. Kannus P, Haapasalo H, Sievanen H et al (1994) The site-specific effects of long-term unilateral activity on bone mineral density and content. Bone 15:279-284

24. Nikander R, Kannus P, Rantalainen T et al (2010) Cross-sectional geometry of weight-bearing tibia in female athletes subjected to different exercise loadings. Osteoporos Int 21:1687-1694

25. von Stengel S, Kemmler W, Kalender WA et al (2007) Differential effects of strength versus power training on bone mineral density in postmenopausal women: a 2-year longitudinal study. Br J Sports Med 41:649-655, discussion 655

26. Martyn-St James M, Carroll S (2009) A meta-analysis of impact exercise on postmenopausal bone loss: the case for mixed loading exercise programmes. Br J Sports Med 43:898-908 